\title{
Ichthyosis and Multiple Sclerosis: A case report
}

\author{
Mehri Salari $^{1}$, Masoud Etemadifar ${ }^{2}$, Sara Ebrahimi $^{2}$, and Amirhossein Akhavan Sigari ${ }^{2}$ \\ ${ }^{1}$ Shahid Beheshti University of Medical Sciences \\ ${ }^{2}$ Isfahan University of Medical Sciences
}

May 14, 2021

\begin{abstract}
Abstract Concurrent presentation of MS and congenital ichthyosis is extremely rare. In this study we present a case of a 27 -year-old woman with congenital ichthyosis who presented with signs of multiple sclerosis. This concurrence may provide insight into any common immunopathologic and genetic mechanisms.
\end{abstract}

\section{Introduction}

Congenital ichthyosis is a heterogeneous group of Mendelian disorders characterized by hyperkeratosis and extensive scaling of the epidermal skin layer due to mutations in genes that play a critical role in epidermal differentiation. Several genetic mutations have been associated with the disease, demonstrating an autosomal recessive, autosomal dominant, and X-linked recessive pattern of inheritance. (1,2) Multiple sclerosis (MS) is an autoimmune inflammatory disease primarily involving the central nervous system, causing variable neurologic manifestations which can ultimately lead to neurologic deterioration. $(3,4)$

In this study, we describe a case of congenital ichthyosis that presented with signs and symptoms of MS to our clinic. Although the co-occurrence of the disease can be a coincidence, the event is uncommon and has been rarely reported.

\section{Case Presentation}

A 27-year-old female with congenital ichthyosis presented to the Isfahan MS clinic with right side vision loss. The decreased vision had started the day before the visit and, upon examination, visual acuity was $4 / 10$ and 9/10 in the right and left eyes, respectively. A grade $2+$ relative afferent pupillary defect was present. She received methylprednisolone $1000 \mathrm{mg}$ daily for three consecutive days which resulted in a major improvement in her right visual acuity $(9 / 10)$. Past medical history was unremarkable. Upon follow up the patient had bilateral upper limb paresthesia five months later. Brain magnetic resonance imaging (MRI) revealed multiple periventricular hyperintensities (figure 1-A). Diagnosis of (MS) was established based on the 2017 revised McDonald criteria (5), and dimethyl fumarate (DMF) was initiated.

The patient was a known case of moderate-severe congenital ichthyosis since birth. The exact type of ichthyosis is not known due to the lack of genetic testing and the patient's missing records from childhood. The diagnosis was made based on clinical grounds and biopsy. She did not have any family history of MS or congenital ichthyosis. The patient is not married and was born full-term from healthy consanguineous parents (the patient's parents are cousins). Physical examination showed extensive scaling and hyperkeratosis of the skin. (figure 1-B,C). The skin was painful in some regions, however, pruritis was not present.

The patient did not have any history of illicit drug or alcohol abuse. She did not take any medications rather than DMF. Complete blood count, liver and thyroid function tests, lipid profile, and creatinine were in the 
normal range. Coagulation factors seven (VII), eight (VIII), nine (IX), and ten (X) were also checked and were normal.

Figure 1 should be inserted here.

\section{Discussion}

Ichthyosis comprises several forms of chronic diseases mainly presenting as extensive scaling, hyperkeratosis, fissuring, and pruritis of the skin. The disease ultimately impairs the protective barrier of the epidermis, mainly the stratum granulosum and stratum corneum layers, leading to the symptoms described above. (6)

Ichthyosis can solely present as skin manifestations (non-syndromic) or along with the involvement of other organs (syndromic). Various genetic mutations have been discovered that contribute to isolated autosomal recessive congenital ichthyosis. (7-10) These can then be phenotypically subdivided into lamellar, erythrodermic, or other forms of ichthyosis. (9)

Moreover, varying neurologic symptoms have been reported with syndromic ichthyosis (neuro-ichthyotic syndromes). (1)

There are few reports of ichthyosis with MS. Capra et al. (12) reports two sisters with congenital ichthyosis and MS back in 1993. One of the cases was diagnosed with MS after lower limb paresthesia and the other experienced right optic neuritis. Both cases were known cases of congenital ichthyosis that later developed MS. They were also found to have coagulation factor VIII deficiency. Although in our case ichthyosis and MS were both present, no other abnormalities such as coagulation factor deficiencies were found.

No underlying genetic correlation between ichthyosis and multiple sclerosis has been elucidated until now. Multiple sclerosis is an autoimmune disease stemming from the interaction of several genetic factors. Therefore, further cases may provide clues to any common genetic links between the two diseases and provide useful guidance regarding disease pathology and effective therapy.

\section{Conclusion}

In this study, we presented a rare case of congenital ichthyosis and MS. Several syndromes of congenital ichthyosis and neurologic manifestations have been identified, however only a few have reported the concurrent presentation of MS with ichthyosis.

\section{Declaration of Interest}

The authors have no conflicts of interest to declare.

\section{Funding}

No funding was received for the provision of this manuscript.

\section{Ethics}

Written consent was obtained from the patient for the presentation of this case.

\section{Consent to Publish}

The patient's consent was taken for the publication of his data in a scientific journal.

\section{Authorship}

MS, ME, and SEP have gathered relevant case information, searched the databases for relevant data, and contributed to the primary manuscript. AAS, SEP, and MS have written the primary draft, revised the manuscript, and searched for relevant data in the medical databases. All authors have read the final manuscript and approved it for publication.

\section{Acknowledgments}


We would like to appreciate the patient's consent as the publication of this article would have not been possible without it.

\section{Ethical approval and consent to participate}

Patient signed the informed consent and accepted the publication of his data for the purpose of publication in a scientific journal. All procedures performed in this study were in accordance with the ethical standards of the institutional research committee and with the 1964 Helsinki declaration and its later amendments or comparable ethical standards.

\section{Data availability statement}

Data sharing is not applicable to this article as no datasets were generated or analyzed during the current study.

\section{References}

1. Vahlquist A, Fischer J, Törmä H. Inherited nonsyndromic ichthyoses: an update on pathophysiology, diagnosis and treatment. Am J Clin Dermatol. 2018;19(1):51-66.

2. Simpson JK, Martinez-Queipo M, Onoufriadis A, Tso S, Glass E, Liu L, et al. Genotype-phenotype correlation in a large English cohort of patients with autosomal recessive ichthyosis. Br J Dermatol. 2020;182(3):729-37.

3. Sigari AA, Etemadifar M, Salari M. Complete horizontal gaze palsy due to bilateral paramedian pontine reticular formation involvement as a presentation of multiple sclerosis: a case report. BMC Neurol. 2019;19(1):1-4.

4. Inojosa H, Schriefer D, Ziemssen T. Clinical outcome measures in multiple sclerosis: A review. Autoimmun Rev [Internet]. 2020;19(5):102512. Available from: https://www.sciencedirect.com/science/article/pii/S1568997220300641

5. Thompson AJ, Banwell BL, Barkhof F, Carroll WM, Coetzee T, Comi G, et al. Diagnosis of multiple sclerosis: 2017 revisions of the McDonald criteria. Lancet Neurol. 2018;17(2):162-73.

6. Diep QM, Luong LH, Tran TH, Dinh OTL, Nguyen HQ, Bui T-H, et al. A case of self-improving collodion ichthyosis in Vietnam. Pediatr Dermatol [Internet]. 2020 May 1;37(3):574-5. Available from: https://doi.org/10.1111/pde.14128

7. Heinz L, Kim G-J, Marrakchi S, Christiansen J, Turki H, Rauschendorf M-A, et al. Mutations in SULT2B1 cause autosomal-recessive congenital ichthyosis in humans. Am J Hum Genet. 2017;100(6):926-39.

8. Boyden LM, Craiglow BG, Hu RH, Zhou J, Browning J, Eichenfield L, et al. Phenotypic spectrum of autosomal recessive congenital ichthyosis due to PNPLA1 mutation. Br J Dermatol. 2017;177(1):319.

9. Youssefian L, Vahidnezhad H, Saeidian AH, Touati A, Sotoudeh S, Mahmoudi H, et al. Autosomal recessive congenital ichthyosis: Genomic landscape and phenotypic spectrum in a cohort of 125 consanguineous families. Hum Mutat. 2019;40(3):288-98.

10. Richard G. Autosomal Recessive Congenital Ichthyosis. 2001 Jan 10 [Updated 2017 May 18]. GeneReviews(r); Adam, MP, Ardinger, HH, Pagon, RA, Wallace, SE, Bean, LJH, Stephens, K, Amemiya, A, Eds. 2018;1993-2020.

11. Rizzo WB, Jenkens SM, Boucher P. Recognition and diagnosis of neuro-ichthyotic syndromes. In: Seminars in neurology. NIH Public Access; 2012. p. 75.

12. Capra R, Mattiolil F, Kalman B, Marciano N, Berenzi A, Benetti A. Two sisters with multiple sclerosis, lamellar ichthyosis, beta thalassaemia minor and a deficiency of factor VIII. J Neurol. 1993;240(6):336-8.

Figure legends 
Figure 1: Figure 1: A- Fluid attenuated inversion recovery (FLAIR) MRI images of the brain showing multiple hyperintensities in the periventricular white matter (Dawson fingers) and juxta cortical areas. B, C- Hyperkeratosis and extensive scaling of the skin.

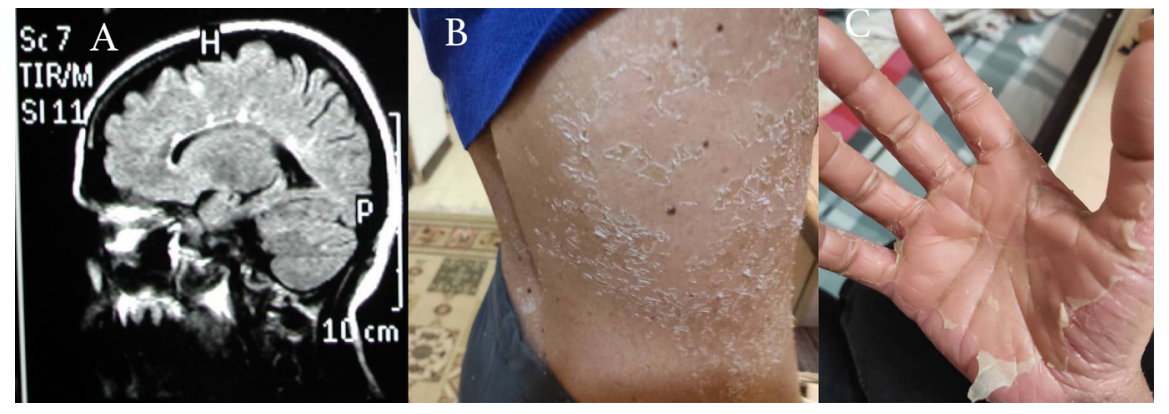

